\title{
Is graphane the most stable carbon monohydride?
}

\author{
M. V. Kondrin, V. V. Brazhkin \\ Institute for High Pressure Physics RAS, 142190 Troitsk, Moscow, Russia \\ mkondrin@hppi.troitsk.ru
}

PACS 61.50.Ah, 61.41.+e, 62.20.D-, 71.15.Mb

DOI $10.17586 / 2220-8054-2016-7-1-44-50$

We discuss a number of hydrocarbon structures whose cohesive energy is not worse than that of benzene and graphanes. These structures can be regarded as sublattices of known carbon structures so the strain exerted on the crystal lattice is minimal and caused mostly by the steric interactions of hydrogen atoms. Possible synthetic routes are proposed. Due to their exceptional mechanical, structural and electrical properties, these crystal structures may have utility as mechanical, optoelectronic or biological materials.

Keywords: graphane,diamond monohydride,hydrocarbons,nitrogen, high pressures.

Received: 20 November 2015

\section{Introduction}

In the recent years, the attention of researchers has been drawn to graphane - carbon monohydride theoretically predicted $[1,2]$ and later experimentally obtained by a complicated synthetic scheme [3]. This compound is interesting due to its exceptional properties (e.g. high temperature superconductivity predicted for properly doped graphane $[4,5]$ ) and as a possible candidate for solid cell hydrogen storage elements [6,7].

However, it should be noted that graphane is not the only compound with a carbonhydrogen ratio of $\mathrm{C}: \mathrm{H}=1: 1$. There are quite a number of other examples: e.g. solid benzene (though at ambient pressure it can exist at low temperatures only [8]), molecular solid phases of cubane/cuneane [9-12] (which can be obtained as metastable forms through complicated organic synthesis), polymeric nanothreads (predicted in [13] and later obtained by high pressure synthesis [14]) and even well-known polysterene. In the case of carbon, there is nothing astonishing that even its metastable forms can have interesting applications. For example, there are at least 4 hypothetical forms [15-17](one of them is similar to the cubic-gauche high pressure phase of nitrogen [18]) that has currently never been observed in practice, but according to quantum chemical calculations are more energetically favorable than actually synthesized cubane. It is interesting to note that according to DFT calculations, graphane $[2,19]$ has the lowest energy among all these allotropes (even lower than benzene).

In this paper, we consider recently proposed 3D hydrocarbon (diamond monohydride/ $\mathrm{DMH}$ ) [20] which can be energetically as viable as graphane. Both can be regarded as sublattices of diamond structure so the internal strain in both is minimal and caused mostly by the steric interactions of hydrogen atoms. We also describe evolution of DMH energy with pressure and discuss its possible analogs among non-organic compounds. 


\section{The least strained structures}

In our previous paper [20], we proposed an heuristic procedure, which can be applied to obtain minimally-strained crystal structures, starting from the known ones (in case of hydrocarbons they are pure carbon structures). To accommodate required number of hydrogen atoms, we need to delete some nodes from the lattice and put hydrogen atom into a bond connecting the deleted node to the nearest carbon atom. From these considerations, restrictions arise. Taking into account relative lengths of $\mathrm{C}-\mathrm{C}$ and $\mathrm{C}-\mathrm{H}$ covalent bonds $(\approx 1.54$ and $\approx 1.0 \AA)$, it is obvious that each unoccupied site can have at most one single occupied node in its vicinity (otherwise the distance between hydrogen atoms on the bonds converging to the same empty node would be too short, resulting in large lattice strain). From this, it immediately follows that the maximum numerical density of structures produced in such a way will be exactly half of the initial amount. Once these conditions are satisfied, it is also clear that the cohesive energies of the proposed structures don't differ much from the initial figures and exact values can be refined subsequently by ab-initio calculations using this postulated structure as a first iteration. However, we would like to stress that even these heuristic considerations will be sufficient to make qualitative predictions about the properties of these structures. We will try to clarify this point in the rest of the paper. Our procedure, to some extent, resembles one adopted previously in the search for zeolite structures [21,22], but we cannot translate it into topological terms of tilings, vertices, etc.

For hydrocarbon structures, one can take diamond and lonsdaleite lattices as starting points. The energy difference between lonsdaleite and diamond is less than $10 \mathrm{meV}$ per bond (it is significantly less than the $20 \mathrm{meV}$ per atom difference between diamond and graphite), so for practical purposes, it can be neglected. There is even a tendency to regard lonsdaleite as a diamond structure, but with a considerable number of stacking faults [23].
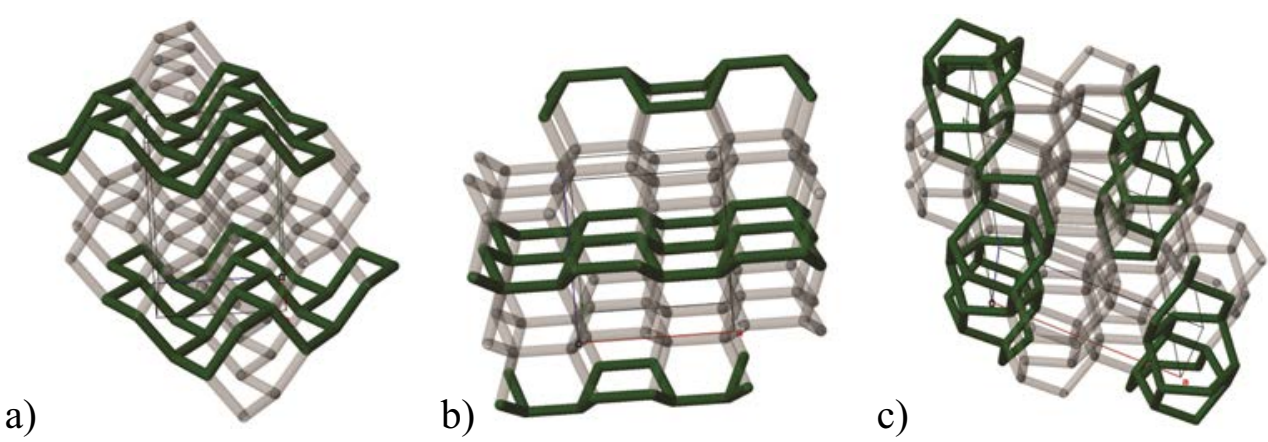

FIG. 1. Diamond-like graphane B (a) and lonsdaleite-like graphane C (b) sheets. c) Hydrocarbon nanothread. Only carbon networks are shown. Translucent bonds demonstrate relations to their respective parent structures

In a more formal way, this procedure can be described as a virtual symmetry breaking operation so the equivalence of lattice nodes in diamond (space group $F d \overline{3} m$, single Wyckoff position $8 a)$ and lonsdaleite $\left(P 6_{3} / m m c, 4 f\right)$ structures aren't retained and their occupancies may vary ${ }^{1}$. Well known graphane structures $[19,29]$ can be described in this way. Depending on the initial structure, they can be classified as either diamond- or lonsdaleite-like. Indeed,

\footnotetext{
${ }^{1}$ For details we refer the reader to the book [24], Bilbao server and programs therein [25-27]. Using crystallographic visualization programs (e.g [28]) tremendously helps in understanding the structural transformations.
} 
virtual phase transitions ${ }^{2} F d \overline{3} m \stackrel{(6,4)}{\longrightarrow}$ Pmna and $F d \overline{3} m \stackrel{(6,4)}{\longrightarrow} P \overline{3} m 1$ lead to splitting $8 a \rightarrow$ $2 \cdot 4 h$ and $\rightarrow 2 \cdot 2 d+1 \cdot 2 c$ respectively which correspond to the formation of two graphane sheets types, named B and A in Ref. [19]. Similarly, from lonsdaleite, graphane structures C (Pmmn subgroup with index 6, 4f $\rightarrow 2 \cdot 4 f)$ and $\mathrm{D}(P b c m$ with index $12, \rightarrow 2 \cdot 8 e$ ) can be obtained. Schematically, relations between 2 types of graphane sheets with their respective parent structures are shown in Fig. 1.

However, in all these cases, the resulting structures are not contiguous; that is, they are not completely covalently bonded. Weak van-der-Waals (vdW) forces allow one to somewhat relax positions of the graphane sheets relative to each other to form more energetically-favorable configurations. For example, shifting of every second sheet of B type into base centered positions results in graphane III structure where carbon atoms form black phosphorus structure. An interesting question arises - is it possible to retain the contiguity of the initial lattice? Though we don't know the general answer, in the particular case of hydrocarbons, the search for an answer leads us to new crystal structures.

For lonsdaleite, it is possible to propose the phase transition with prime klassengleiche index $7 P 6_{3} / m m c \stackrel{(2,7)}{\longrightarrow} P 6_{3} / m$ which corresponds to the expansion of the unit cell in two dimensions along the basal plane. This transition leads to splitting $4 f \rightarrow 2 \cdot 12 i+1 \cdot 4 f$, producing a structure (Fig. $1 \mathrm{c}$ ) which can be described as a nanothread [13,14] bundle.

\section{DMH structure and its properties}

It is remarkable that though nanothread bundle is $\mathrm{vdW}$ bonded structure but the procedure similar to described above in case of diamond produces completely covalently bonded DMH crystal. Formally, this is described as a $F d \overline{3} m \stackrel{(8,14)}{\longrightarrow} R \overline{3}$ transition accompanied by splitting $8 a \rightarrow 2 \cdot 6 c+4 \cdot 18 f$ (in our previous work [20] we have overlooked rhombohedral symmetry). Each of positions' sets can be evenly distributed between occupied and unoccupied nodes. So this structure can be regarded as a result of "halving" of the diamond one. This also dictates the parameters for the resulting conventional unit cell (obtuse hexagonal with $a \approx 6.67$ $\AA, c \approx 12.36 \AA$ and $Z=42$ formula units in it).

As an initial estimate for structure optimization, hydrogen atoms are put into this structure $1 \AA$ apart from occupied sites in the direction pointing to the closest unoccupied one (see Fig. 2). The resulting structure turns out to be more energetically favorable than benzene and comparable to diamond-like graphanes. For details of DFT calculation using QuantumESPRESSO software [30] we refer the reader to [20]. Evolution of the energy difference between DMH and graphane with pressure is shown in Fig. 3.

Because carbon atoms in $\mathrm{DMH}$ are in the $s p^{3}$-hybridized state, it is not surprising that the substance is good dielectric with quite a large band gap of $4.5 \mathrm{eV}$ (comparable to the theoretical diamond's one but direct). DMH density is somewhat less than half that of diamond's (due to $5 \%$ expansion of covalent bonds) and equals $1.7 \mathrm{~g} / \mathrm{cm}^{3}$. Because DMH is related to parent structure through removal of some $\mathrm{C}-\mathrm{C}$ bonds, the mechanical properties are significantly inferior to that of diamond, but still quite impressive (bulk and shear moduli are $B=90 \pm 15$ and $G=60 \pm 15 \mathrm{GPa}$ respectively). One remarkable DMH feature is the presence of quite spacious "tubes" piercing the bulk of the structure (see Fig. 2). As it was pointed

\footnotetext{
${ }^{2}$ The numbers above arrows designate translationengleiche and klassengleiche subgroup indices $t, k$. In general such a transition leads to splitting of Wyckoff positions of parent structure into several sets of Wyckoff positions of subgroup. Such a relation we will designate by the notation $4 f \rightarrow 2 \cdot 2 d+1 \cdot 2 c$, meaning that $8 a$ Wyckoff position of the parent group is split into two sets of $2 d$ and one set of $2 c$ subgroup. The left part can be omitted if it is clear which position is meant.
} 
a)

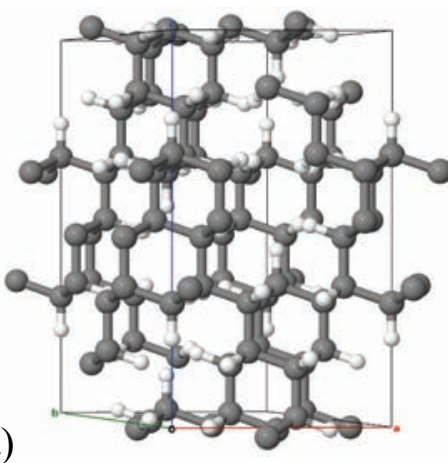

b)

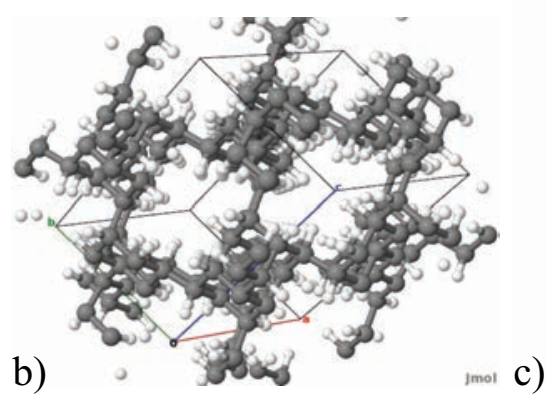

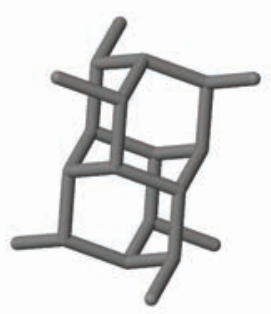

c)

FIG. 2. Structure of DMH. Perspective view along base plane (a) and along pores (b). Grey spheres stands for carbon atoms and white for hydrogen. c) The building block of DMH structure - diamantane molecule with six loose bonds (only carbon framework is depicted)
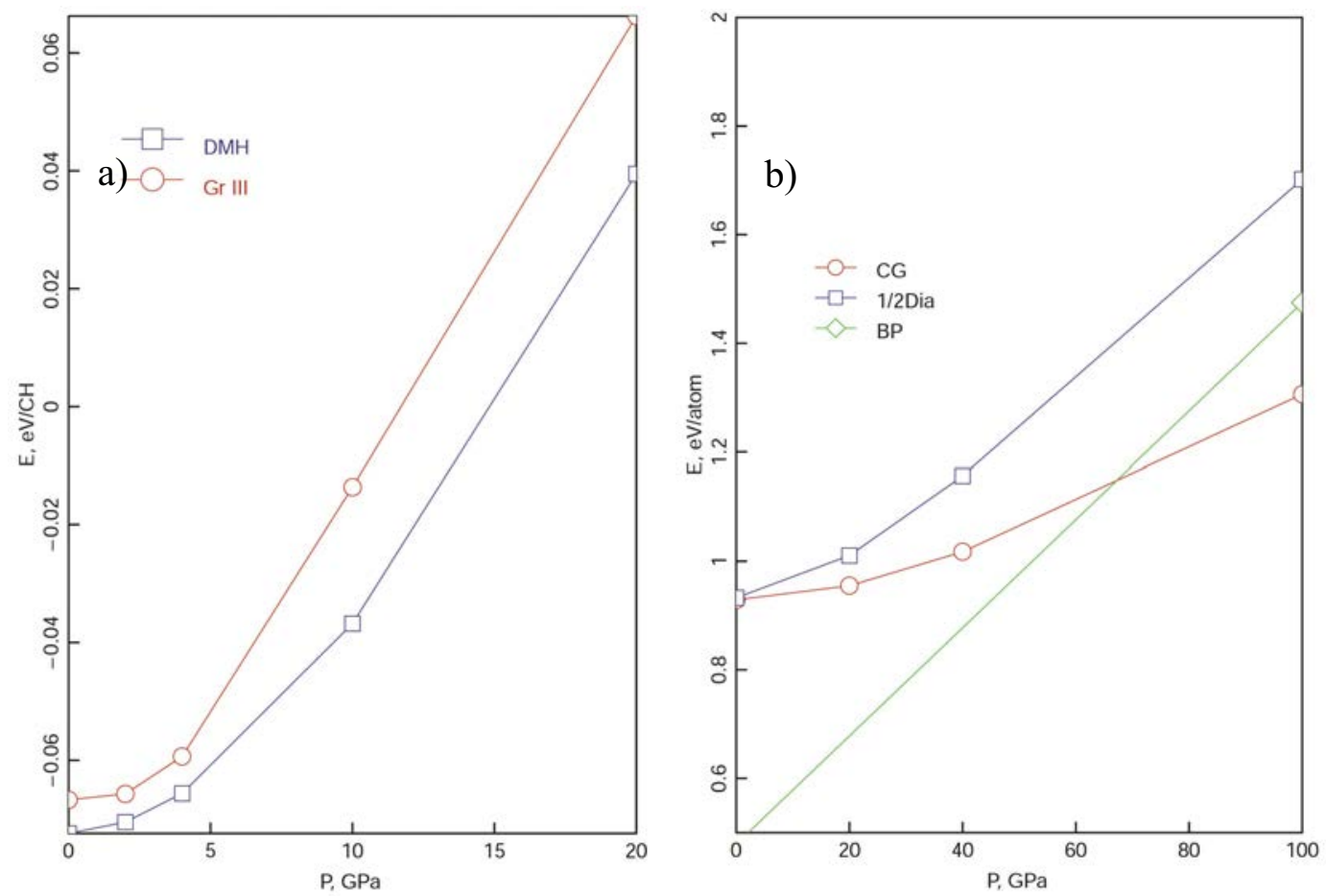

FIG. 3. Pressure evolution of energy in a) hydrocarbon structures $(0-$ graphane III and $\square-\mathrm{DMH}$ ) and b) nitrogen ( $\circ-$ cubic gauche, $\diamond-$ black phosphorus and $\square$ - "half-diamond" or DMH-like). In both cases the energy of respective molecular phase (benzene and $\mathrm{N}_{2}$ ) at ambient pressure is taken as zero

out in Ref. [31] in the context of carbon-nitrogen compounds the filling of such pores with metallic atoms would lead to almost one dimensional metallic conductivity which in certain circumstances (e.g. strong interaction with phonones of hydrocarbon matrix) could produce high-temperature superconductivity.

Though at first glance, the crystal structure seems extremely complicated, but in reality,this is not true. In fact, it can be thought of as an ordered network of polymerized diamantane molecules $\left(\mathrm{C}_{14} \mathrm{H}_{20}\right)$ [32] with six hydrogen atoms removed. So the building block of this structure is a diamantane molecule with six dangling bonds (Fig. 2). Its point group is $\overline{3}$, so naturally 
it can be packed in $R \overline{3}$ space group. The diamantane molecule is quite rigid and the DMH structure's unit cell consists of six molecules of such geometry that they are related to each other by crystallographic symmetry.

This observation also gives a clue which possibly will help to produce DMH using properly functionalized/substituted diamantane molecules (this is somewhat similar to considerations present in Refs. [33,34] but there, adamantane molecules were used as building blocks). If it would be possible to attach some sort of radicals $X$ to secondary carbon atoms in diamantane molecules, such that the hypothetical chemical dimerization $\left(2 \cdot{ }_{14} H_{14} X_{6} \rightarrow\right.$ $2 \cdot\left(C_{14} H_{14} X_{5}\right)+2 \cdot X_{2}$ is possible, then the self-organization of these molecules into a crystal structure can be expected.

\section{Possible non-organic analogues}

Because the DMH structure cannot be readily found in nature (in contrast to benzene and diamantanes which can be separated from the crude oil) it would be worthwhile to look for possible analogs of the DMH structure in the inorganic realm. One possible candidate would be binary sphalerite compounds which are widely used in electronics. For compounds with high enough ionicity (like GaSb or better), a certain reordering of atoms can be expected, which would lead to arrangement with one type of atoms taking positions of occupied nodes in DMH structure while others take positions of empty ones. However, we are not aware of any finding of this kind [35].

Another possibility is the exploiting of the analog between $\mathrm{CH}$ radical and group five elements (where a fully occupied orbital plays the role of a $\mathrm{C}-\mathrm{H}$ bond). In fact, there much similarity between them, for example, in graphane I and III structures, carbon atoms form a network similar to arsenic and black phosphorus structures. As a model object, the nitrogen structures can be extremely educative. For example, the structure of nitrogen at pressures higher than $100 \mathrm{GPa}$ can be considered as "halving" of bC8 structure (observed in the wild in $\mathrm{Si}, \mathrm{Ge}$ [35], presumably produced under certain conditions in vapor deposited carbon [36] and predicted to be thermodynamically stable in pure carbon at TPa pressures [37]). Here, the symmetry breaking is quite simple and consists of the removal of an inversion center, thus

producing a virtual transition $I a \overline{3} \stackrel{2,1}{\longrightarrow} I 2_{1} 3$. The resulting enantiomorphic crystal structure in the context of nitrogen [38] was named cubic gauche (however one may expect droite conformation too) and was considered previously as a possible candidate for 3D hydrocarbon structures [15]. Although the corresponding hydrocarbon structure significantly loses to DMH and graphanes (about than $0.2 \mathrm{eV}$ per $\mathrm{CH}$ group), in the case of nitrogen, the situation is inverted. The relative energies of cubic gauche, DMH-like and black phosphorus type nitrogen crystal structures are depicted in Fig. 3. The behavior of bp- and cg-N generally coincides with the results of Ref. [38] but the energy of DMH-like nitrogen is comparable with that of cg-N only in the low density region and with increased pressure, this difference rapidly increases. It should also be noted that the pressure region in Fig. 3 below $50 \mathrm{GPa}$ has no practical significance because there, molecular nitrogen with triple covalent bond is the unquestionable winner.

Such a difference between DMH and its nitrogen analog is possibly caused by the effective charge of $\mathrm{C}-\mathrm{H}$ group on the one hand and filled electron orbital in nitrogen on the other. The higher coulomb interaction in nitrogen favors its crystallization in the form where two types of bonds exist (similar to bC8 structure) so the diamond and lonsdaleite structures (where all bonds are equivalent) are simply bad initial approximations. 


\section{Conclusion}

The main conclusion of this paper is fairly obvious and was determined many times before $[39,40]$, however, it is worth repeating once more. That is, the energy considerations are not the decisive factor in the question of viability of some compound (especially an organic one) but the availability of technological process for its fabrication is. The examples considered in this paper include cubane (it even loses to the cubic gauche hydrocarbon structure) and nanothreads, which are of the lonsdaleite type, and such, lose to diamond-type graphanes but nonetheless can be obtained by the high-pressure high-temperature treatment of molecular benzene [14]. A similar consideration applied to DMH structure implies that its manufacturing requires some preliminary steps involving production of suitable precursors. However these efforts may be worthwhile because of its exceptional mechanical, structural and electrical properties. DMH might have utility as a material for biological and optical applications or as a solid hydrogen storage element.

\section{Acknowledgements}

The authors acknowledge financial support from the RSF (Grant No. 14-22-00093) and thank Y. B. Lebed for assistance and helpful discussions.

\section{References}

[1] Sluiter M. H. F., Kawazoe Y. Cluster expansion method for adsorption: Application to hydrogen chemisorption on graphene. Phys. Rev. B, 2003, 68 (8), P. 085410.

[2] Sofo J., Chaudhari A., Barber G. Graphane: A two-dimensional hydrocarbon. Phys. Rev. B, 2007,75 (15), P. 153401.

[3] Elias D. C., Nair R. R., Mohiuddin T. M. G., Morozov S. V., Blake P., Halsall M. P., Ferrari A. C., Boukhvalov D. W., Katsnelson M. I., Geim A. K., Novoselov K. S. Control of Graphene's Properties by Reversible Hydrogenation: Evidence for Graphane. Science, 2009, 323 (5914), P. 610-613.

[4] Savini G., Ferrari A. C., Giustino F. First-Principles Prediction of Doped Graphane as a High-Temperature Electron-Phonon Superconductor. Phys. Rev. Lett., 2010, 105 (3), P. 037002.

[5] Loktev V., Turkowski V. Possible High-Temperature Superconductivity in Multilayer Graphane: Can the Cuprates be Beaten? Journal of Low Temperature Physics, 2011, 164 (5-6), P. 264-271.

[6] Peng Q., Dearden A. K., Crean J., Han L., Liu S., Wen X., De S. New materials graphyne, graphdiyne, graphone, and graphane: review of properties, synthesis, and application in nanotechnology. Nanotechnology, Science and Applications, 2104, 7, P. 1-29.

[7] Zhou C., Chen S., Lou J., Wang J., Yang Q., Liu C., Huang D., Zhu T. Graphene's cousin: the present and future of graphane. Nanoscale Research Letters, 2014, 9 (1), P. 26.

[8] Ciabini L., Gorelli F. A., Santoro M., Bini R., Schettino V., Mezouar M. High-pressure and high-temperature equation of state and phase diagram of solid benzene. Phys. Rev. B, 2005, 72 (9), P. 094108.

[9] Eaton P. E., Cole T. W. Cubane. Journal of the American Chemical Society, 1964, 86 (15), P. $3157-3158$.

[10] Fleischer E. B. X-Ray Structure Determination of Cubane. Journal of the American Chemical Society, 1964, 86 (18), P. 3889-3890.

[11] Yildirim T., Gehring P., Neumann D., Eaton P., Emrick T. Unusual structure, phase transition, and dynamics of solid cubane. Phys. Rev. Lett., 1997, 78 (26), P. 4938-4941.

[12] Criegee R., Askani R. Octamethylsemibullvalene. Angewandte Chemie International Edition in English, 1968, 7 (7), P. 537-537.

[13] Wen X.-D., Hoffmann R., Ashcroft N. W. Benzene under High Pressure: a Story of Molecular Crystals Transforming to Saturated Networks, with a Possible Intermediate Metallic Phase. Journal of the American Chemical Society, 2011, 133 (23), P. 9023-9035.

[14] Fitzgibbons T. C., Guthrie M., shi Xu E., Crespi V. H., Davidowski S. K., Cody G. D., Alem N., Badding J. V. Benzene-derived carbon nanothreads. Nature Materials, 2015, 14, P. 43-47.

[15] Lian C.-S., Wang X.-Q., Wang J.-T. Hydrogenated K4 carbon: A new stable cubic gauche structure of carbon hydride. The Journal of Chemical Physics, 2013, 138 (2), P. 024702. 
[16] He C., Sun L. Z., Zhang C. X., Zhong J. Low energy three-dimensional hydrocarbon crystal from cold compression of benzene. Journal of Physics: Condensed Matter, 2013, 25 (20), P. 205403.

[17] Lian C.-S., Li H.-D., Wang J.-T. Crystalline structures of polymeric hydrocarbon with 3,4-fold helical chains. Sci. Rep., 2015, 5, P. 07723.

[18] Eremets M. I., Gavriliuk A. G., Trojan I. A., Dzivenko D. A., Boehler R. Single-bonded cubic form of nitrogen. Nat Mater, 2004, 3 (8), P. 558-563.

[19] Wen X.-D., Hand L., Labet V., Yang T., Hoffmann R., Ashcroft N. W., Oganov A. R., Lyakhov A. O. Graphane sheets and crystals under pressure. Proceedings of the National Academy of Sciences, 2011, 108 (17), P. 68336837.

[20] Kondrin M. V., Brazhkin V. V. Diamond monohydride: the most stable three-dimensional hydrocarbon. Phys. Chem. Chem. Phys., 2015, 17 (27), P. 17739-17744.

[21] Blatov V. A., Proserpio D. M. Periodic-graph approaches in crystal structure prediction. In Modern Methods of Crystal Structure Prediction (Oganov A. R., editor), chapter 1, pp. 1-28. Wiley-VCH Verlag GmbH \& Co. $\mathrm{KGaA}, 2010$.

[22] Delgado-Friedrichs O., Foster M. D., O’Keeffe M., Proserpio D. M., Treacy M. M. J., Yaghi O. M. What do we know about three-periodic nets? Journal of Solid State Chemistry, 2005, 178 (8), P. 2533 - 2554.

[23] Németh P., Garvie L. A. J., Aoki T., Dubrovinskaia N., Dubrovinsky L., Buseck P. R. Lonsdaleite is faulted and twinned cubic diamond and does not exist as a discrete material. Nat Commun, 2014, 5.

[24] Tolèdano P., Dmitriev V. Reconstructive phase transitions in crystals and quasicrystals. World Scientific, Singapore, 1996.

[25] Aroyo M. I., Perez-Mato J. M., Capillas C., Kroumova E., Ivantchev S., Madariaga G., Kirov A., Wondratschek H. Bilbao Crystallographic Server: I. Databases and crystallographic computing programs. Zeitschrift für Kristallographie, 2006, 221 (1), P. 15-27.

[26] Capillas C., Kroumova E., Aroyo M. I., Perez-Mato J. M., Stokes H. T., Hatch D. M. SYMMODES: a software package for group-theoretical analysis of structural phase transitions. Journal of Applied Crystallography, 2003, 36 (3 Part 2), P. 953-954.

[27] Kroumova E., Perez-Mato J. M., Aroyo M. I. WYCKSPLIT: a computer program for determination of the relations of Wyckoff positions for a group-subgroup pair. J. Appl. Cryst., 1998, 31, P. 646.

[28] Jmol: an open-source Java viewer for chemical structures in 3D. http://www.jmol.org/.

[29] Artyukhov V. I., Chernozatonskii L. A. Structure and Layer Interaction in Carbon Monofluoride and Graphane: A Comparative Computational Study. The Journal of Physical Chemistry A, 2010, 114 (16), P. 5389-5396.

[30] Giannozzi P., Baroni S., et al . QUANTUM ESPRESSO: a modular and open-source software project for quantum simulations of materials. Journal of Physics: Condensed Matter, 2009, 21 (39), P. 395502.

[31] Balaban A. T., Klein D. J., Seitz W. A. Holes in diamond or carbon nitride lattices. International Journal of Quantum Chemistry, 1996, 60 (5), P. 1065-1068.

[32] Clay W. A., Dahl J. E. P., Carlson R. M. K., Melosh N. A., Shen Z.-X. Physical properties of materials derived from diamondoid molecules. Reports on Progress in Physics, 2015, 78 (1), P. 016501.

[33] Garcia J. C., Assali L. V. C., Machado W. V. M., Justo J. F. Crystal engineering using functionalized adamantane. Journal of Physics: Condensed Matter, 2010, 22 (31), P. 315303.

[34] Garcia J. C., Justo J. F., Machado W. V. M., Assali L. V. C. Functionalized adamantane: Building blocks for nanostructure self-assembly. Phys. Rev. B, 2009, 80 (12), P. 125421.

[35] Mujica A., Rubio A., Muñoz A., Needs R. J. High-pressure phases of group-IV, III-V, and II-VI compounds. Rev. Mod. Phys., 2003, 75 (3), P. 863-912.

[36] Johnston R. L., Hoffmann R. Superdense carbon, $\mathrm{C}_{8}$ : supercubane or analog of $\gamma$-silicon? Journal of the American Chemical Society, 1989, 111 (3), P. 810-819.

[37] Correa A. A., Bonev S. A., Galli G. Carbon under extreme conditions: Phase boundaries and electronic properties from first-principles theory. Proceedings of the National Academy of Sciences, 2006, 103 (5), P. 1204-1208.

[38] Mailhiot C., Yang L. H., McMahan A. K. Polymeric nitrogen. Phys. Rev. B, 1992, 46 (22), P. 14419-14435.

[39] Brazhkin V. V. Metastable phases, phase transformations, and phase diagrams in physics and chemistry. Physics-Uspekhi, 2006, 49 (7), P. 719.

[40] Brazhkin V. V. Interparticle interaction in condensed media: some elements are 'more equal than others'. Physics-Uspekhi, 2009, 52 (4), P. 369. 